\begin{tabular}{|c|l|}
\hline Title & Catalytic Enantioselective A ziridination of A lkenes U sing Chiral Dirhodium(II) Carboxylates \\
\hline Author(s) & Y amawaki, Minoru; Tanaka, Masahiko; A be, Takumi; A nada, Masahiro; Hashimoto, Shunichi \\
\hline Citation & Heterocycles- An International Journal for Reviews and Communications in Heterocyclic Chemistry, 72, 709-721 \\
\hline Issue Date & 2007-04-13 \\
\hline Doc URL & http://hdl.handle.net/2115/26427 \\
\hline Type & article (author version) \\
\hline File Information & HE72-709.pdf \\
\hline
\end{tabular}

Instructions for use 
HETEROCYCLES, Vol. , No. , , pp. -. ( ) The Japan Institute of Heterocyclic Chemistry

Received, , Accepted, , Published online, . COM-06- (Please do not delete.)

\title{
CATALYTIC ENANTIOSELECTIVE AZIRIDINATION OF ALKENES USING CHIRAL DIRHODIUM(II) CARBOXYLATES ${ }^{\dagger}$
}

\author{
Minoru Yamawaki, Masahiko Tanaka, Takumi Abe, Masahiro Anada, and \\ Shunichi Hashimoto* \\ Faculty of Pharmaceutical Sciences, Hokkaido University, Sapporo 060-0812, \\ Japan \\ e-mail: hsmt@pharm.hokdudai.ac.jp
}

\begin{abstract}
The enantioselective aziridination of alkenes with [ $N$-(4-nitrophenylsulfonyl) imino]phenyliodinane catalyzed by dirhodium(II) tetrakis[ $N$-tetrachlorophthaloyl-(S)-tert-leucinate], $\mathrm{Rh}_{2}(S \text {-TCPTTL })_{4}$, is described. While such enantioselectivities are highly dependent on the properties of the alkenes, 2,2-dimethylchromene was found to be a particularly suitable substrate which can be efficiently transformed into the aziridine product in $98 \%$ yield with $94 \%$ ee.
\end{abstract}

The enantioselective nitrene transfer reaction from [ $N$-(arylsulfonyl)imino]phenyliodinanes to alkenes catalyzed by chiral transition metal complexes represents one of the most direct and powerful methods for the construction of optically active aziridines which are versatile building blocks for the synthesis of biologically important, nitrogen-containing molecules. ${ }^{1}$ Over the past fifteen years, substantial progress has been made in the development of enantioselective variants through catalysis by copper, ${ }^{2-4}$ manganese, ${ }^{5,6}$ and ruthenium ${ }^{7}$ complexes of well-designed chiral ligands, in which excellent levels of enantioselectivity have been achieved with a limited range of alkenes. ${ }^{8}$

While the first use of $\mathrm{Rh}_{2}(\mathrm{OAc})_{4}$ as a nitrene transfer catalyst was demonstrated by Breslow and Gellman in $1983,{ }^{9}$ Evans and co-workers in the early 1990s reported that $\mathrm{Cu}(\mathrm{I})$ and $\mathrm{Cu}(\mathrm{II})$ triflate and perchlorate salts were far superior to $\mathrm{Rh}_{2}(\mathrm{OAc})_{4}$ in aziridination reactions using [N-(4-methyphenylsulfony)imino]phenyliodinane. ${ }^{10}$ Thus, the application of chiral dirhodium(II) complexes to enantioselective aziridination has been less investigatated. In 1996, Müller and co-workers demonstrated the first example of the dirhodium(II)-catalyzed enantioselective aziridination of styrene and cis- $\beta$-methylstyrene (up to $73 \%$ ee) by exploiting [ $N$-(4-nitrophenylsulfonyl)imino]phenyliodinane

$\dagger$ Dedicated to Professor Yoshito Kishi on the occasion of his 70th birthday. 
(2a) as a nitrene precursor and dirhodium(II) binaphtholphosphate complex $\operatorname{Rh}_{2}(R-\mathrm{BNP})_{4}(\mathbf{1 d})$ as a chiral catalyst. ${ }^{11}$ Thereafter, the Che, Müller, and Hayes groups independently reported enantioselective intramolecular aziridinations of sulfonamides, sulfamate esters, and carbamates via in situ generated iminoiodinanes in the presence of chiral dirhodium(II) carboxamidate catalysts, $\operatorname{Rh}_{2}(4 S \text {-MEOX })_{4}(\mathbf{1 e})$ and $\mathrm{Rh}_{2}(5 S-\mathrm{MEPY})_{4}$ (1f), and dirhodium(II) prolinate catalyst $\mathrm{Rh}_{2}(S \text {-TBSP })_{4}$ (1g), in which cyclic sulfonamides, ${ }^{12 \mathrm{a}, \mathrm{b}}$ cyclic sulfamidates, ${ }^{12 \mathrm{c}}$ and oxazolidinones ${ }^{12 \mathrm{~d}}$ were obtained in up to $76 \%, 52 \%$, and $23 \%$ ee, respectively.

We recently demonstrated that the enantioselective benzylic $\mathrm{C}-\mathrm{H}$ amidation of aromatic hydrocarbons with [ $N$-(4-nitrophenylsulfonyl)imino]phenyliodinane (2a) catalyzed by chiral dirhodium(II) carboxylates provides sulfonamides in up to $84 \%$ ee [eqn. (1)]. ${ }^{13}$ In this process, $\operatorname{Rh}_{2}(S \text {-TCPTTL })_{4}(\mathbf{1 a})$, characterized by the substitution of chlorine atoms for four hydrogen atoms on the phthalimido group in the parent dirhodium(II) complex, $\mathrm{Rh}_{2}(S \text {-PTTL })_{4}(\mathbf{1 c}),{ }^{14,15}$ proved to be the catalyst of choice in terms of product yield and enantioselectivity as well as catalytic activity. Very recently, we also reported on the one-pot enantioselective intramolecular $\mathrm{C}-\mathrm{H}$ amidation of sulfamate esters using $\mathrm{Rh}_{2}(S \text {-TFPTTL })_{4}(\mathbf{1 b})$ as a chiral catalyst and $\mathrm{PhI}(\mathrm{OAc})_{2}$ as an oxidant, in which cyclic sulfamidates were obtained in up to $48 \%$ ee [eqn. (2)]. ${ }^{16}$ As a logical extension of our studies, we now address the issue of enantiocontrol in the intermolecular aziridination of alkenes.
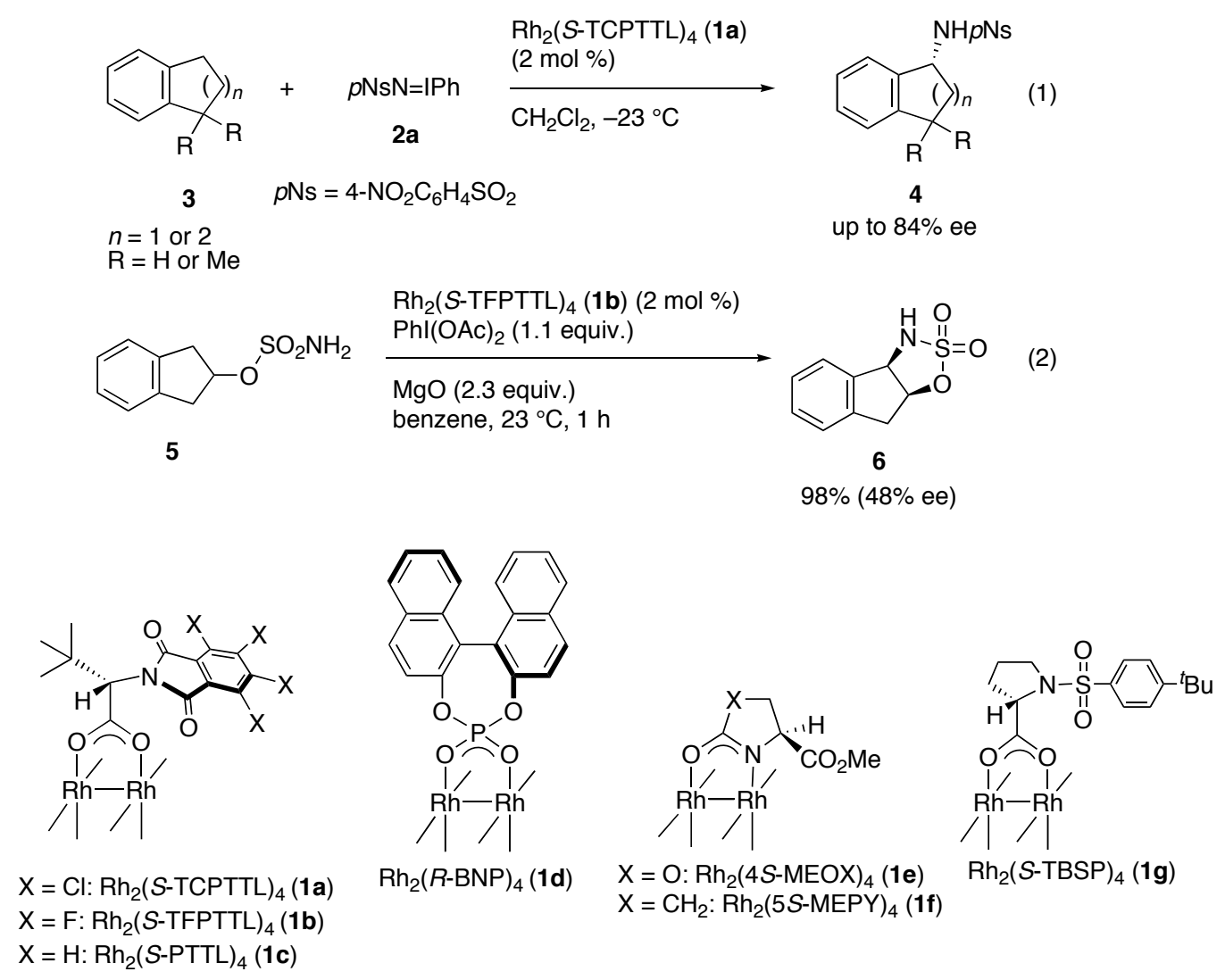

At the outset, we explored the aziridination of styrene (7a) with 1.1 equiv. of $2 \mathbf{a}$ in the presence of 2 mol \% of $\mathrm{Rh}_{2}(\mathrm{~S} \text {-TCPTTL })_{4}(\mathbf{1 a})$. In dichloromethane at $0{ }^{\circ} \mathrm{C}$, the reaction proceeded smoothly to give 
Table 1. Enantioselective Aziridination of Styrene (7a) Catalyzed by Chiral Dirhodium(II) Carboxylates $^{\text {a) }}$

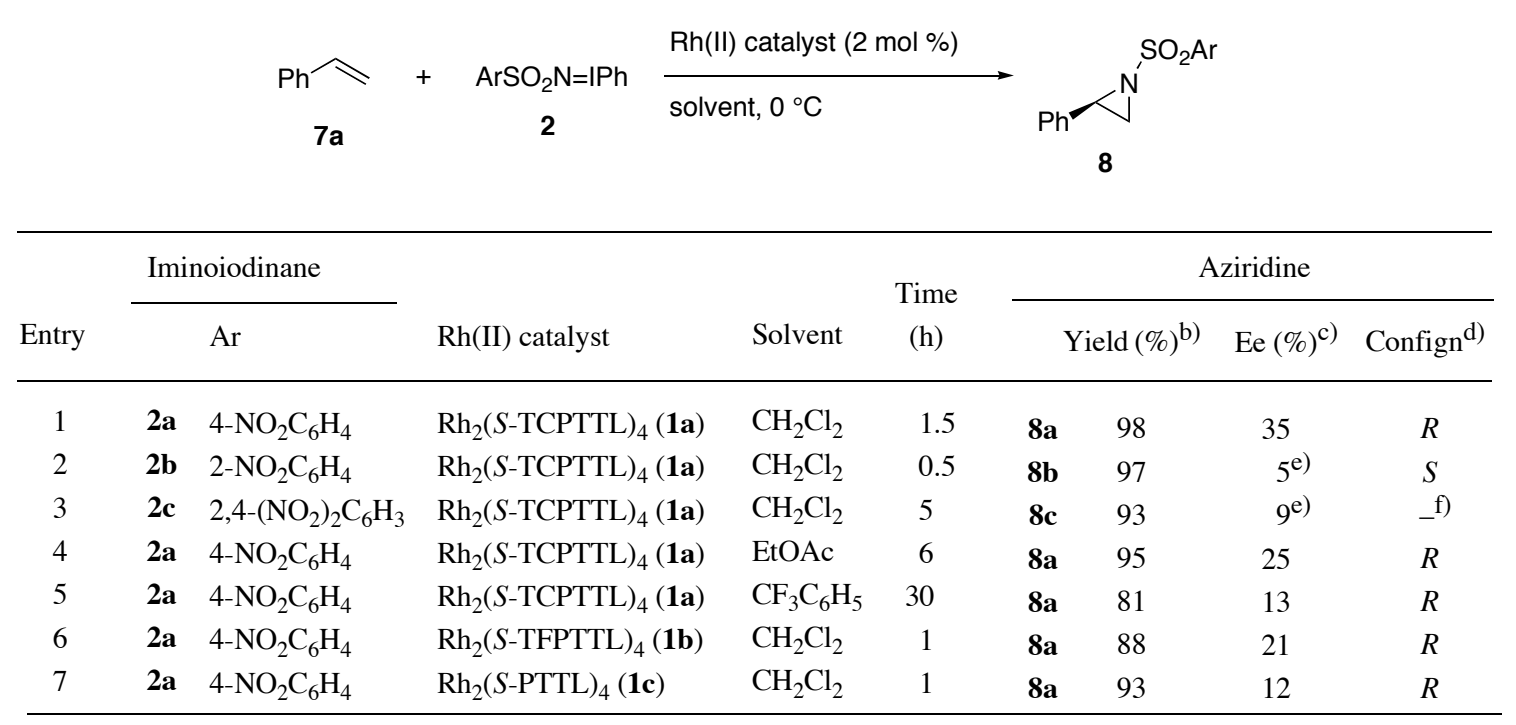

a) All reactions were performed on a 0.2 mmol scale. ${ }^{\text {b) }}$ Isolated yield. ${ }^{c)}$ Determined by HPLC (Daicel Chiralpak AD-H column) unless otherwise stated. d) Determined by comparison of the sign of optical rotation with the lierature value.

e) Determined by HPLC (Daicel Chiralpak AD column). ${ }^{\text {f) }}$ Absolute configuration was not determined.

aziridine $(\mathbf{8 a}),[\alpha]_{\mathrm{D}}^{23}-24.2^{\circ}\left(c 1.14, \mathrm{CHCl}_{3}\right)$, in $98 \%$ yield (Table 1, Entry 1$)$. The enantioselectivity of this reaction was determined to be $35 \%$ ee by HPLC analysis (Daicel Chiralpak AD-H). The preferred absolute configuration of $\mathbf{8 a}$ was established as $R$ by comparing the sign of the optical rotation with the literature value $\left[\right.$ lit., ${ }^{17}[\alpha]_{\mathrm{D}}+77.8^{\circ}\left(c \quad 1.0, \mathrm{CHCl}_{3}\right)$ for $(S)$-8a $]$. Although the use of [ $N$-(2-nitrophenylsulfonyl)imino]phenyliodinane (2b) and [N-(2,4-dinitrophenylsulfonyl)imino]phenyliodinane (2c) as nitrene precursors provided the corresponding aziridines $(\mathbf{8 b}, \mathbf{c})$ in high yield, the enantioselectivity was significantly diminished (Entries 2 and 3). ${ }^{18}$ A survey of solvents revealed that dichloromethane was the optimal solvent for this reaction. The use of ethyl acetate provided $(R)-8 \mathbf{a}$ in 95\% yield with $25 \%$ ee, but the reaction required much longer times to reach completion (Entry 4). Benzotrifluoride was found to be the least effective in terms of both reaction rate and enantioselectivity (Entry 5). We then evaluated the performance of two other chiral dirhodium(II) complexes, $\mathrm{Rh}_{2}(S \text {-TFPTTL })_{4} \quad(\mathbf{1 b})^{19}$ and $\mathrm{Rh}_{2}(S \text {-PTTL })_{4} \quad(\mathbf{1 c}){ }^{14,15}$ derived from $N$-tetrafluorophthaloyl- and $N$-phthaloyl-(S)-tert-leucine, respectively. Although both catalysts $(\mathbf{1 b}, \mathbf{c})$ afforded $(R)-\mathbf{8 a}$ at similar reaction rates and yields as those found with $1 \mathbf{1 a}, \mathrm{Rh}_{2}(S \text {-TCPTTL })_{4}$ proved to be the catalyst of choice for producing a more reasonable degree of enantioselectivity (Entries 1 vs. 6 and 7).

With optimized conditions in hand, we then investigated the applicability of the present catalytic system to alkenes other than styrene. Some representative results are presented in Table 2. Styrene derivatives (7b-d) with electron-withdrawing groups at the para position on the benzene ring exhibited higher enantioselectivities than styrene (58\%, $40 \%$ and $36 \%$ ee, Entries 1-3). In contrast, a sharp drop in 
Table 2. Enantioselective Aziridination of Alkenes with 3a Catalyzed by $\mathrm{Rh}_{2}(S \text {-TCPTTL })_{4}(\mathbf{1 a})^{\mathrm{a})}$

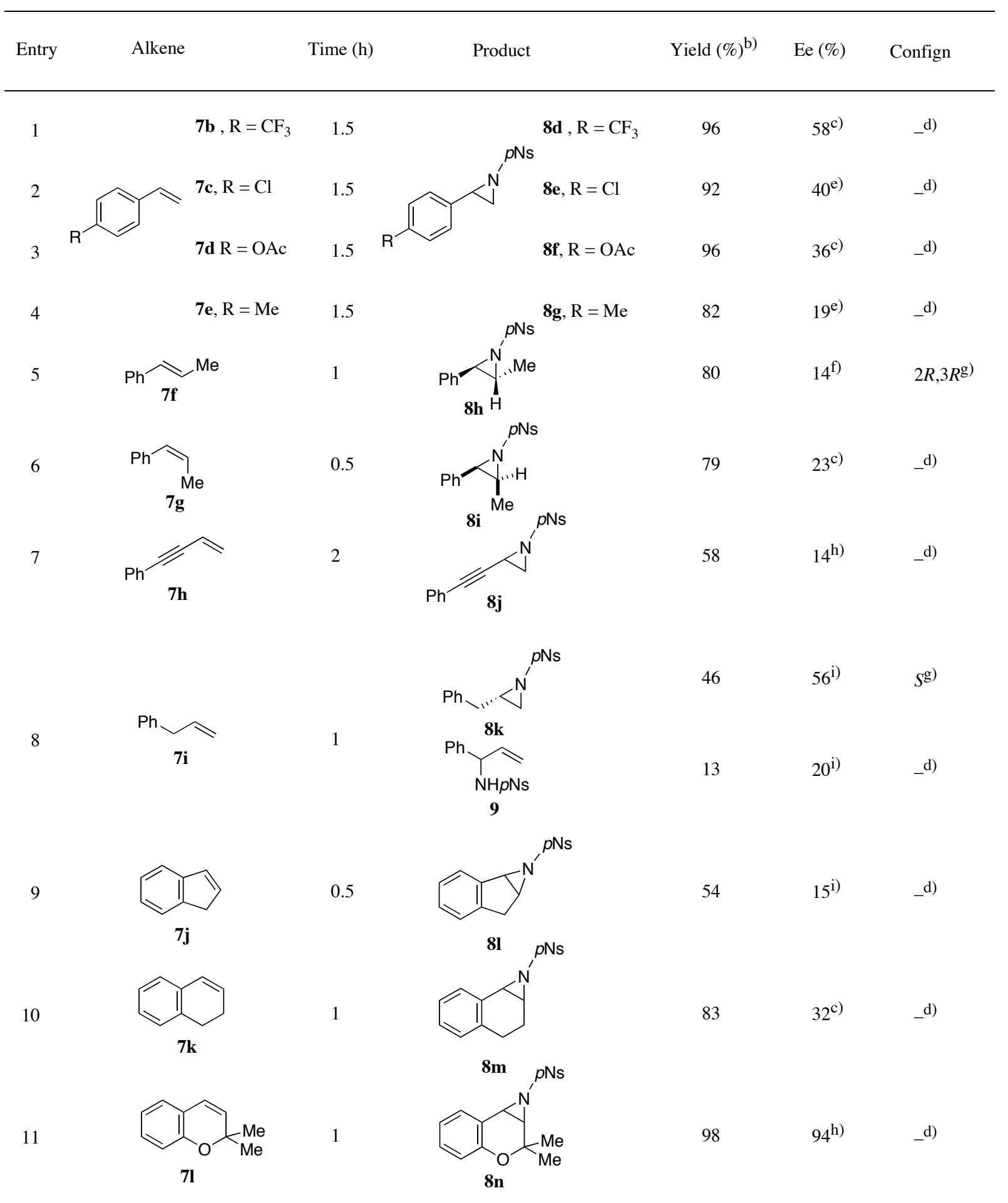

a) All reactions were performed at $0{ }^{\circ} \mathrm{C}$ using $2 \mathrm{~mol} \%$ of Rh(II) catalyst and 1.1 equiv. of 2a . ${ }^{\text {b) }}$ Isolated yield. ${ }^{\text {c) }}$ Determined by HPLC (Daicel Chiralpak AS-H column). ${ }^{\text {d) }}$ Absolute configuration was not determined. ${ }^{\text {e) }}$ Determined by HPLC (Daicel Chiralpak AS column). f) Determined by HPLC (Daicel Chiralpak AD column $\times 2$ ). ${ }^{\mathrm{g})}$ Determined by comparison of optical rotation with the literature value.

h) Determined by HPLC (Daicel Chiralcel OD-H column). ${ }^{\text {i) }}$ Determined by HPLC (Daicel Chiralpak AD-H column).

enantioselectivity was observed when 4-methylstyrene (7e), which contains an electron-donating substituent, was used (19\% ee, Entry 4). As expected from results for the $\mathrm{Rh}_{2}(\mathrm{OAc})_{4}$-catalyzed aziridination reported by Müller, ${ }^{11}$ the reactions of trans- and cis- $\beta$-methylstyrene (7f,g) with $2 \mathbf{2 a}$ proceeded in a stereospecific manner to give the derived trans- and cis-2,3-disubstituted aziridines $(\mathbf{8 h}, \mathbf{i})$ 
in high yields, although the ee values were significantly lower than that found with 7a (Entries 5 and 6). The aziridination of the alkyne-conjugated terminal alkene (7h) also resulted in a low enantioselectivity (Entry 7). On the other hand, the reaction with allylbenzene (7i) provided the aziridine (8k) in $46 \%$ yield with $56 \%$ ee (Entry 8 ). The low yield in this reaction was due to the competitive formation of the allylic $\mathrm{C}-\mathrm{H}$ amidation product $(\mathbf{9})^{20}(13 \%$ yield, $20 \%$ ee). The aziridination of indene $(\mathbf{7 j})$ and 1,2-dihydronaphthalene (7k) resulted in $15 \%$ and 32\% ee, respectively (Entries 9 and 10). It is interesting to note that no allylic or benzylic $\mathrm{C}-\mathrm{H}$ amidation products were obtained in these reactions. Gratifyingly, the use of 2,2-dimethylchromene (7l) produced the aziridine (8n) in $98 \%$ yield with $94 \%$ ee (Entry 11). While exceptionally high levels of enantioselectivity (>98\% ee) in the aziridination of 6-substituted 2,2-dimethylchromenes have already been achieved using the copper(I)-diimine complexes pioneered by Jacobsen, ${ }^{3 \mathrm{a}, \mathrm{d}, \mathrm{g}, \mathrm{i}}$ the enantioselectivity of $94 \%$ ee obtained for $\mathbf{1 a}$ is the highest reported to date for a chiral dirhodium(II) complex-catalyzed aziridination reaction.

In summary, we have reported the enantioselective aziridination of alkenes with [ $N$-(4-nitrophenylsulfonyl)imino]phenyliodinane using $\mathrm{Rh}_{2}(S \text {-TCPTTL })_{4}$ as a catalyst. Only in the case of 2,2-dimethylchromene, a high level of enantioselectivity ( $94 \%$ ee) has been achieved. The design and synthesis of a new class of chiral dirhodium(II) catalysts to extend the scope of such enantioselective nitrene transfer reactions is currently in progress.

\section{EXPERIMENTAL}

General. Melting points were determined on a Büchi 535 digital melting point apparatus and are uncorrected. IR spectra were recorded on a JASCO FT/IR-4100 spectrometer and absorbance bands are reported in wavenumber $\left(\mathrm{cm}^{-1}\right)$. ${ }^{1} \mathrm{H}$ NMR spectra were recorded on JEOL JNM-ECX400P (400 MHz) spectrometer. Chemical shifts are reported relative to internal standard (tetramethylsilane; $\delta_{\mathrm{H}} 0.00$ ). Data are presented as follows: chemical shift $(\delta, \mathrm{ppm})$, multiplicity $(\mathrm{s}=$ singlet, $\mathrm{d}=$ doublet, $\mathrm{t}=$ triplet, $\mathrm{q}=$ quartet, $\mathrm{m}=$ multiplet), coupling constant and integration. ${ }^{13} \mathrm{C}$ NMR spectra were recorded on JEOL JNM-ECX400P (100 MHz) spectrometer. Chemical shifts are reported relative to internal standard $\left(\mathrm{CDCl}_{3} ; \delta 77.0\right)$. Optical rotations were measured on a JASCO P-1030 digital polarimeter at the sodium D line $(589 \mathrm{~nm})$. EI-MS spectra were obtained on a JEOL JMS-HX 110 spectrometer. Column chromatography was carried out on Wakogel ${ }^{\circledR}$ C-200 (75-150 $\left.\mu \mathrm{m}\right)$. Analytical thin layer chromatography (TLC) was carried out on Merck Kieselgel $60 \mathrm{~F}_{254}$ plates with visualization by ultraviolet, anisaldehyde stain solution or phosphomolybdic acid stain solution. Analytical high performance liquid chromatography (HPLC) was performed on JASCO PU-980 intelligent HPLC pump with JASCO UV-970 intelligent UV/VIS detector. Detection was performed at $254 \mathrm{~nm}$. Chiralpak AD, AD-H, AS, 
AS-H and Chiralcel OD-H columns $(0.46 \mathrm{~cm} \times 25 \mathrm{~cm})$ from Daicel were used. Retention times $\left(t_{\mathrm{R}}\right)$ and peak ratio were determined with JASCO-Borwin analysis system.

All reactions were carried out in flame-dried glassware under argon atmosphere unless otherwise noted. Reagents and solvents were purified by standard means. [ $N$-(arylsulfonyl)imino]phenyliodinanes (2a-c) were prepared from corresponding sulfonamides according to the literature procedure. ${ }^{21}$

General procedure for the enantioselective intermolecular aziridination reaction (Table 1, Entry 1):

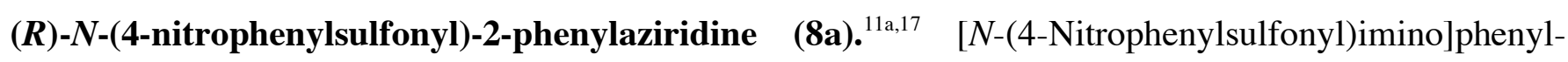
iodinane (2a) $(88.9 \mathrm{mg}, 0.22 \mathrm{mmol}, 1.1$ equiv.) was added in one portion to a solution of styrene (7a) (20.8 mg, $0.20 \mathrm{mmol})$ and bis(ethyl acetate) adduct of $\mathrm{Rh}_{2}(S$-TCPTTL) 4 (7.9 mg, $0.004 \mathrm{mmol}, 2 \mathrm{~mol} \%)$ in $\mathrm{CH}_{2} \mathrm{Cl}_{2}(2 \mathrm{~mL})$ at $0{ }^{\circ} \mathrm{C}$. After $1.5 \mathrm{~h}$ of stirring at this temperature, the whole mixture was concentrated in vacuo and purified by column chromatography (Wakogel ${ }^{\circledR} \mathrm{C}-200,9: 1$ hexane/EtOAc) to provide $(R)-8 \mathbf{a}(59.6 \mathrm{mg}, 98 \%)$ as a white solid; $R_{f}=0.34\left(3: 1\right.$ hexane/EtOAc); mp $131.5-134.0{ }^{\circ} \mathrm{C} ;[\alpha]_{\mathrm{D}}{ }^{23}-24.2^{\circ}$ $\left(c 1.14, \mathrm{CHCl}_{3}\right)$ for $35 \%$ ee $\left[\right.$ lit., ${ }^{17}[\alpha]_{\mathrm{D}}+77.8^{\circ}\left(c 1.0, \mathrm{CHCl}_{3}\right)$ for $\left.(S)-8 \mathrm{a}\right] ;{ }^{1} \mathrm{H} \mathrm{NMR}\left(400 \mathrm{MHz}, \mathrm{CDCl}_{3}\right) \delta$ $2.51(\mathrm{~d}, J=4.5 \mathrm{~Hz}, 1 \mathrm{H}, \mathrm{NCH} H), 3.12(\mathrm{~d}, J=7.2 \mathrm{~Hz}, 1 \mathrm{H}, \mathrm{NCHH}), 3.91(\mathrm{dd}, J=4.5,7.2 \mathrm{~Hz}, 1 \mathrm{H}, \mathrm{NCHPh})$, 7.22-7.23 (m, 2H, Ar), 7.30-7.34 (m, 2H, Ar), 8.19 (d, J = 9.1 Hz, 2H, Ar), 8.38 (d, J = 9.1 Hz, 2H, Ar). The enantiomeric excess of $\mathbf{8 a}$ was determined to be $35 \%$ by HPLC with a Chiralpak AD-H column (9:1 hexane $/ i$-PrOH, $1.0 \mathrm{~mL} / \mathrm{min}$ ): $t_{\mathrm{R}}$ (major) $=25.9 \min$ for $(R)$-enantiomer; $t_{\mathrm{R}}$ (minor) $=29.1 \mathrm{~min}$ for $(S)$-enantiomer.

(S)-N-(2-Nitrophenylsulfonyl)-2-phenylaziridine $(\mathbf{8 b}){ }^{22}$ According to the general procedure for enantioselective aziridination reaction, $8 \mathbf{b}$ was prepared from $7 \mathbf{a}(20.8 \mathrm{mg}, 0.20 \mathrm{mmol})$ and [ $N$-(2-nitrophenylsulfonyl)imino]phenyliodinane (2b) (88.9 mg, $0.22 \mathrm{mmol}, 1.1$ equiv.). The crude product was purified by column chromatography (Wakogel ${ }^{\circledR}$ C-200, 9:1 hexane/EtOAc) to provide $(S)-8 b$ $(58.4 \mathrm{mg}, 97 \%)$ as a yellow oil; $R_{f}=0.37\left(3: 1\right.$ hexane/EtOAc); $[\alpha]_{\mathrm{D}}{ }^{17}+4.90^{\circ}\left(c 1.42, \mathrm{CHCl}_{3}\right)$ for $5 \%$ ee $\left[\right.$ lit., ${ }^{22}[\alpha]_{\mathrm{D}}{ }^{25}-99.7^{\circ}\left(\mathrm{c} 1.0, \mathrm{CHCl}_{3}\right)$ for $\left.(R)-8 \mathrm{~b}\right] ;{ }^{1} \mathrm{H} \mathrm{NMR}\left(400 \mathrm{MHz}, \mathrm{CDCl}_{3}\right) \delta 2.63(\mathrm{~d}, J=4.8 \mathrm{~Hz}, 1 \mathrm{H}$, $\mathrm{NCH} H), 3.24(\mathrm{~d}, J=7.3 \mathrm{~Hz}, 1 \mathrm{H}, \mathrm{NCHH}), 4.03(\mathrm{dd}, J=4.8,7.3 \mathrm{~Hz}, \mathrm{NCHPh}), 7.28-7.36(\mathrm{~m}, 5 \mathrm{H}, A r)$, 7.70-7.36 (m, 3H, Ar), 8.21-8.24 (m, 1H, Ar). The enantiomeric excess of $\mathbf{8 b}$ was determined to be $5 \%$ by HPLC with a Chiralpak AD column $\left(5: 1\right.$ hexane $/ i-\mathrm{PrOH}, 1.0 \mathrm{~mL} / \mathrm{min}$ ): $t_{\mathrm{R}}$ (major) $=16.6 \mathrm{~min}$ for $(S)$-enantiomer; $t_{\mathrm{R}}$ (minor) $=20.3 \min$ for $(R)$-enantiomer.

$N$-(2,4-Dinitrophenylsulfonyl)-2-phenylaziridine $(\mathbf{8 c}){ }^{11 \mathrm{~b}}$ According to the general procedure for enantioselective aziridination reaction, 8c was prepared from 7a $(20.8 \mathrm{mg}, 0.20 \mathrm{mmol})$ and [ $N$-(2,4-dinitrophenylsulfonyl)imino]phenyliodinane (2c) $(98.8 \mathrm{mg}, 0.22 \mathrm{mmol}, 1.1$ equiv.). The crude 
product was purified by column chromatography (Wakoge ${ }^{\circledR} \mathrm{C}-200,5: 1$ hexane/EtOAc) to provide 8c $(64.6 \mathrm{mg}, 93 \%)$ as a yellow oil; $R_{f}=0.28\left(3: 1\right.$ hexane/EtOAc); $[\alpha]_{\mathrm{D}}{ }^{19}+5.13^{\circ}\left(c 1.92, \mathrm{CHCl}_{3}\right)$ for $9 \%$ ee; ${ }^{1} \mathrm{H}$ NMR $\left(400 \mathrm{MHz}, \mathrm{CDCl}_{3}\right) \delta 2.71(\mathrm{~d}, J=4.8 \mathrm{~Hz}, 1 \mathrm{H}, \mathrm{NCH} H), 3.32(\mathrm{~d}, J=7.1 \mathrm{~Hz}, 1 \mathrm{H}, \mathrm{NCH} H), 4.11$ (dd, $J=4.8,7.1 \mathrm{~Hz}, \operatorname{ArCH}), 7.26-7.34(\mathrm{~m}, 5 \mathrm{H}, A r), 8.45-8.47$ (m, 1H, Ar), 8.53-8.56 (m, 2H, Ar). The enantiomeric excess of $\mathbf{8 c}$ was determined to be $9 \%$ by HPLC with a Chiralpak AD column (3:1 hexane $/ i-\mathrm{PrOH}, 2.0 \mathrm{~mL} / \mathrm{min}$ ): $t_{\mathrm{R}}$ (major) $=10.2 \mathrm{~min} ; t_{\mathrm{R}}($ minor $)=20.3 \mathrm{~min}$. The absolute configuration of 8c was not determined.

$N$-(4-Nitrophenylsulfonyl)-2-[(4-trifluoromethyl)phenyl]aziridine (8d). ${ }^{23}$ According to the general procedure for enantioselective aziridination reaction, 8d was prepared from 4-(trifluoromethyl)styrene (7b) (34.4 $\mathrm{mg}, 0.2 \mathrm{mmol})$ and $\mathbf{2 a}(88.9 \mathrm{mg}, 0.22 \mathrm{mmol}, 1.1$ equiv.). The crude product was purified by column chromatography (Wakogel ${ }^{\circledR}$ C-200, 9:1 hexane/EtOAc) to provide 8d $(71.3 \mathrm{mg}, 96 \%$ ) as a colorless oil; $R_{f}=0.35$ (3:1 hexane/EtOAc); $[\alpha]_{\mathrm{D}}{ }^{23}-30.6^{\circ}\left(c 1.10, \mathrm{CHCl}_{3}\right)$ for $58 \%$ ee; ${ }^{1} \mathrm{H} \mathrm{NMR}(400$ $\left.\mathrm{MHz}, \mathrm{CDCl}_{3}\right) \delta 2.49$ (d, $\left.J=4.6 \mathrm{~Hz}, 1 \mathrm{H}, \mathrm{NCH} H\right), 3.15$ (d, $\left.J=7.4 \mathrm{~Hz}, 1 \mathrm{H}, \mathrm{NCH} H\right), 3.95$ (dd, $J=4.6,7.4$ $\mathrm{Hz}, 1 \mathrm{H}, \operatorname{ArCH}), 7.36$ (d, $J=8.2 \mathrm{~Hz}, 2 \mathrm{H}, A r), 7.59$ (d, $J=8.2 \mathrm{~Hz}, 2 \mathrm{H}, A r), 8.20$ (d, $J=9.1 \mathrm{~Hz}, 2 \mathrm{H}, A r)$, $8.41(\mathrm{~d}, J=9.1 \mathrm{~Hz}, 2 \mathrm{H}, A r)$. The enantiomeric excess of $\mathbf{8 d}$ was determined to be $58 \%$ by HPLC with a Chiralpak AS-H column (3:1 hexane $/ i-\mathrm{PrOH}, 2.0 \mathrm{~mL} / \mathrm{min}): t_{\mathrm{R}}$ (major) $=8.9 \mathrm{~min} ; t_{\mathrm{R}}($ minor $)=14.8 \mathrm{~min}$. The absolute configuration of $\mathbf{8 d}$ was not determined.

2-(4-Chlorophenyl)- $N$-(4-nitrophenylsulfonyl)aziridine (8e). ${ }^{23}$ According to the general procedure for enantioselective aziridination reaction, 8e was prepared from 4-chlorostyrene (7c) $(27.7 \mathrm{mg}, 0.2 \mathrm{mmol})$ and $2 \mathbf{a}(88.9 \mathrm{mg}, 0.22 \mathrm{mmol}, 1.1$ equiv.). The crude product was purified by column chromatography (Wakogel ${ }^{\circledR}$ C-200, 9:1 hexane/EtOAc) to provide $8 \mathbf{e}(61.5 \mathrm{mg}, 92 \%)$ as a colorless solid; $R_{f}=0.39(3: 1$ hexane/EtOAc); mp 97.0-98.5 ${ }^{\circ} \mathrm{C} ;[\alpha]_{\mathrm{D}}^{23}-25.7^{\circ}\left(c\right.$ 1.46, $\left.\mathrm{CHCl}_{3}\right)$ for $40 \%$ ee; ${ }^{1} \mathrm{H} \mathrm{NMR}\left(400 \mathrm{MHz}, \mathrm{CDCl}_{3}\right)$ $\delta 2.47(\mathrm{~d}, J=4.5 \mathrm{~Hz}, 1 \mathrm{H}, \mathrm{NCH} H), 3.11(\mathrm{~d}, J=7.2 \mathrm{~Hz}, 1 \mathrm{H}, \mathrm{NCHH}), 3.88(\mathrm{dd}, J=4.5,7.2 \mathrm{~Hz}, \operatorname{ArCH})$, $7.16(\mathrm{~d}, J=8.9 \mathrm{~Hz}, 2 \mathrm{H}, A r), 7.29(\mathrm{~d}, J=8.9 \mathrm{~Hz}, 2 \mathrm{H}, A r), 8.18(\mathrm{~d}, J=9.1 \mathrm{~Hz}, 2 \mathrm{H}, A r), 8.39(\mathrm{~d}, J=9.1 \mathrm{~Hz}$, $2 \mathrm{H}, A r)$. The enantiomeric excess of $8 \mathrm{e}$ was determined to be $40 \%$ by HPLC with a Chiralpak AS column $(3: 1$ hexane $/ i-\mathrm{PrOH}, 2.0 \mathrm{~mL} / \mathrm{min}): t_{\mathrm{R}}$ (major) $=13.9 \mathrm{~min} ; t_{\mathrm{R}}$ (minor) $=22.0 \mathrm{~min}$. The absolute configuration of $\mathbf{8 e}$ was not determined.

2-(4-Acetoxyphenyl)- $N$-(4-nitrophenylsulfonyl)aziridine (8f). ${ }^{11 \mathrm{~b}}$ According to the general procedure for enantioselective aziridination reaction, $8 \mathbf{f}$ was prepared from 4-acetoxystyrene (7d) (32.4 $\mathrm{mg}, 0.2 \mathrm{mmol})$ and $2 \mathbf{a}(88.9 \mathrm{mg}, 0.22 \mathrm{mmol}, 1.1$ equiv.). The crude product was purified by column chromatography (Wakogel $^{\circledR}$ C-200, 4:1 hexane/EtOAc) to provide $8 f(69.4 \mathrm{mg}, 96 \%)$ as a white solid; $R_{f}=0.29(2: 1$ 
hexane/EtOAc); mp 143.0-144.0 ${ }^{\circ} \mathrm{C} ;[\alpha]_{\mathrm{D}}{ }^{23}-21.4^{\circ}\left(c 0.91, \mathrm{CHCl}_{3}\right)$ for $36 \%$ ee; ${ }^{1} \mathrm{H} \mathrm{NMR}(400 \mathrm{MHz}$, $\left.\mathrm{CDCl}_{3}\right) \delta 2.29$ (s, 3H, $\left.\mathrm{CH}_{3} \mathrm{CO}\right), 2.48$ (d, $\left.J=4.6 \mathrm{~Hz}, 1 \mathrm{H}, \mathrm{NCH} H\right), 3.11$ (d, $\left.J=7.3 \mathrm{~Hz}, 1 \mathrm{H}, \mathrm{NCHH}\right), 3.90$ $(\mathrm{dd}, J=4.6,7.3 \mathrm{~Hz}, \operatorname{ArCH}), 7.04(\mathrm{~d}, J=8.7 \mathrm{~Hz}, 2 \mathrm{H}, A r), 7.24(\mathrm{~d}, J=8.7 \mathrm{~Hz}, 2 \mathrm{H}, A r), 8.19(\mathrm{~d}, J=9.2 \mathrm{~Hz}$, $2 \mathrm{H}, A r), 8.40(\mathrm{~d}, J=9.2 \mathrm{~Hz}, 2 \mathrm{H}, A r)$. The enantiomeric excess of $\mathbf{8 f}$ was determined to be $36 \%$ by HPLC with a Chiralpak AS-H column $(1: 1$ hexane $/ i-\mathrm{PrOH}, 2.0 \mathrm{~mL} / \mathrm{min}): t_{\mathrm{R}}($ major $)=12.7 \mathrm{~min} ; t_{\mathrm{R}}($ minor $)=20.0$ min. The absolute configuration of $\mathbf{8 f}$ was not determined.

2-(4-Methylphenyl)- $N$-(4-nitrophenylsulfonyl)aziridine $(8 \mathrm{~g}) .{ }^{11 a}$ According to the general procedure for enantioselective aziridination reaction, $\mathbf{8 g}$ was prepared from 4-methylstyrene (7e) (23.6 $\mathrm{mg}, 0.2 \mathrm{mmol})$ and $2 \mathbf{a}(88.9 \mathrm{mg}, 0.22 \mathrm{mmol}, 1.1$ equiv.). The crude product was purified by column chromatography (Wakogel ${ }^{\circledR}$ C-200, 9:1 hexane/EtOAc) to provide $8 \mathrm{~g}(51.7 \mathrm{mg}, 82 \%)$ as a white solid; $R_{f}=0.47$ (3:1 hexane/EtOAc); mp 139.0-140.0 ${ }^{\circ} \mathrm{C} ;[\alpha]_{\mathrm{D}}{ }^{23}-12.7^{\circ}\left(c 0.83, \mathrm{CHCl}_{3}\right.$ ) for $19 \%$ ee; ${ }^{1} \mathrm{H} \mathrm{NMR}(400 \mathrm{MHz}$, $\left.\mathrm{CDCl}_{3}\right) \delta 2.32\left(\mathrm{~s}, 3 \mathrm{H}, \mathrm{ArCH}_{3}\right), 2.50(\mathrm{~d}, J=4.6 \mathrm{~Hz}, 1 \mathrm{H}, \mathrm{NCHH}), 3.10(\mathrm{~d}, J=7.2 \mathrm{~Hz}, 1 \mathrm{H}, \mathrm{NCH} H), 3.86$ $(\mathrm{dd}, J=4.6,7.2,1 \mathrm{H}, \operatorname{ArCH}), 7.08-7.13(\mathrm{~m}, 4 \mathrm{H}, A r), 8.18(\mathrm{~d}, J=8.8 \mathrm{~Hz}, 2 \mathrm{H}, A r), 8.37$ (d, $J=8.8 \mathrm{~Hz}, 2 \mathrm{H}$, $A r)$. The enantiomeric excess of $\mathbf{8 g}$ was determined to be $19 \%$ by HPLC with a Chiralpak AS column $(3: 1$ hexane $/ i-\mathrm{PrOH}, 1.0 \mathrm{~mL} / \mathrm{min}): t_{\mathrm{R}}$ (major) $=19.1 \mathrm{~min} ; t_{\mathrm{R}}$ (minor) $=27.1 \mathrm{~min}$. The absolute configuration of $\mathbf{8 g}$ was not determined.

(2R,3R)-2-Methyl- $N$-(4-nitrophenylsulfonyl)-3-phenylaziridine $(8 \mathrm{~h}) .^{2 \mathrm{~d}}$ According to the general procedure for enantioselective aziridination reaction, $\mathbf{8 h}$ was prepared from trans- $\beta$-methylstyrene (7f) (23.6 $\mathrm{mg}, 0.2 \mathrm{mmol})$ and $\mathbf{2 a}(88.9 \mathrm{mg}, 0.22 \mathrm{mmol}, 1.1$ equiv.). The crude product was purified by column chromatography (Wakogel ${ }^{\circledR} \mathrm{C}-200,9: 1$ hexane/EtOAc) to provide $(2 R, 3 R)-8 \mathbf{h}(50.7 \mathrm{mg}, 80 \%)$ as a pale yellow oil; $R_{f}=0.40\left(3: 1\right.$ hexane/EtOAc); $[\alpha]_{\mathrm{D}}{ }^{23}-3.93^{\circ}\left(c 0.83, \mathrm{CHCl}_{3}\right)$ for $14 \%$ ee $\left[\right.$ lit. ${ }^{2 \mathrm{~d}}[\alpha]_{\mathrm{D}}{ }^{24}+60.4^{\circ}(\mathrm{c}$ $\left.1.08, \mathrm{CHCl}_{3}\right)$ for $80 \%$ ee of $\left.(2 S, 3 S)-8 \mathrm{~h}\right] ;{ }^{1} \mathrm{H} \mathrm{NMR}\left(400 \mathrm{MHz}, \mathrm{CDCl}_{3}\right) \delta 1.89\left(\mathrm{~d}, J=6.3 \mathrm{~Hz}, 3 \mathrm{H}, \mathrm{CH}_{3}\right)$, 3.05 (dq, $J=4.5,6.3 \mathrm{~Hz}, 1 \mathrm{H}, \mathrm{CH}_{3} \mathrm{CH}$ ), 3.87 (d, $J=4.5 \mathrm{~Hz}, \mathrm{PhCH}$ ), 7.12-7.15 (m, 2H, Ar), 7.26-7.29 (m, $3 \mathrm{H}, A r), 8.12(\mathrm{~d}, J=9.1 \mathrm{~Hz}, 2 \mathrm{H}, A r), 8.30(\mathrm{~d}, J=9.1 \mathrm{~Hz}, 2 \mathrm{H}, A r)$. The enantiomeric excess of $\mathbf{8 h}$ was determined to be $14 \%$ by HPLC with a Chiralpak AD $(\times 2)$ columns $(9: 1$ hexane $/ i-\mathrm{PrOH}, 1.0 \mathrm{~mL} / \mathrm{min}): t_{\mathrm{R}}$ $($ major $)=16.6 \min$ for $(2 R, 3 R)$-enantiomer; $t_{\mathrm{R}}($ minor $)=33.4 \min$ for $(2 S, 3 S)$-enantiomer.

cis-2-Methyl- $N$-(4-nitrophenylsulfonyl)-3-phenylaziridine (8i). ${ }^{11 a}$ According to the general procedure for enantioselective aziridination reaction, 8i was prepared from cis- $\beta$-methylstyrene (7g) (23.6 mg, 0.2 mmol) and 2a (88.9 mg, $0.22 \mathrm{mmol}, 1.1$ equiv.). The crude product was purified by column chromatography (Wakogel ${ }^{\circledR} \mathrm{C}-200,9: 1$ hexane/EtOAc) to provide 8i (49.7 mg, 79\%) as a pale yellow oil; $R_{f}=0.42\left(3: 1\right.$ hexane/EtOAc); $[\alpha]_{\mathrm{D}}{ }^{23}+18.3^{\circ}\left(c 1.19, \mathrm{CHCl}_{3}\right)$ for $23 \%$ ee; ${ }^{1} \mathrm{H} \mathrm{NMR}\left(400 \mathrm{MHz}, \mathrm{CDCl}_{3}\right) \delta$ 
$1.06\left(\mathrm{~d}, J=5.9 \mathrm{~Hz}, 3 \mathrm{H}, \mathrm{CH}_{3}\right), 3.34\left(\mathrm{dq}, J=7.2,5.9 \mathrm{~Hz}, 1 \mathrm{H}, \mathrm{CH}_{3} \mathrm{CH}\right), 4.08(\mathrm{~d}, J=7.2 \mathrm{~Hz}, \mathrm{PhCH})$, 7.18-7.21 (m, 2H, Ar), 7.29-7.31 (m, 3H, Ar), 8.22 (d, J=8.6 Hz, 2H, Ar), 8.39 (d, J = 8.6 Hz, 2H, Ar). The enantiomeric excess of $\mathbf{8 i}$ was determined to be $23 \%$ by HPLC with a Chiralpak AS-H column (9:1 hexane $/ i-\mathrm{PrOH}, 2.0 \mathrm{~mL} / \mathrm{min}): t_{\mathrm{R}}($ major $)=18.2 \mathrm{~min} ; t_{\mathrm{R}}($ minor $)=22.0 \mathrm{~min}$. The absolute configuration of 8i was not determined.

$N$-(4-Nitrophenylsulfonyl)-2-(2-phenylethynyl)aziridine (8j). ${ }^{8 \mathrm{~b}}$ According to the general procedure for enantioselective aziridination reaction, $\mathbf{8 j}$ was prepared from (3-buten-1-ynyl)benzene (7h) (25.6 mg, 0.2 mmol) and 2a (88.9 $\mathrm{mg}, 0.22 \mathrm{mmol}, 1.1$ equiv.). The crude product was purified by column chromatography (Wakogel ${ }^{\circledR}$ C-200, 9:1 hexane/EtOAc) to provide $8 \mathbf{j}$ (37.5 mg, 58\%) as a pale yellow oil; $R_{f}=0.42\left(3: 1\right.$ hexane/EtOAc); $[\alpha]_{\mathrm{D}}{ }^{26}-5.30^{\circ}\left(c 0.69, \mathrm{CHCl}_{3}\right)$ for $14 \%$ ee; ${ }^{1} \mathrm{H} \mathrm{NMR}\left(400 \mathrm{MHz}, \mathrm{CDCl}_{3}\right) \delta$ $2.63(\mathrm{~d}, J=4.5 \mathrm{~Hz}, 1 \mathrm{H}, \mathrm{NCH} H), 2.99(\mathrm{~d}, J=7.2 \mathrm{~Hz}, 1 \mathrm{H}, \mathrm{NCHH}), 3.60(\mathrm{dd}, J=4.5,7.2 \mathrm{~Hz}, \mathrm{C} \equiv \mathrm{CCH})$, 7.26-7.41 (m, 4H, Ar), $8.21(\mathrm{~d}, J=9.1 \mathrm{~Hz}, 2 \mathrm{H}, A r), 8.42$ (d, $J=9.1 \mathrm{~Hz}, 2 \mathrm{H}, A r)$. The enantiomeric excess of $\mathbf{8 j}$ was determined to be $14 \%$ by HPLC with a Chiralcel OD-H column $(3: 1$ hexane/i-PrOH, 1.0 $\mathrm{mL} / \mathrm{min}): t_{\mathrm{R}}($ major $)=28.5 \mathrm{~min} ; t_{\mathrm{R}}($ minor $)=38.6 \mathrm{~min}$. The absolute configuration of $8 \mathbf{j}$ was not determined.

(S)-2-Benzyl- $N$-(4-nitrophenylsulfonyl)aziridine $(\mathbf{8 k}) .^{24}$ According to the general procedure for enantioselective aziridination reaction, 8k was prepared from allylbenzene (7i) (23.6 $\mathrm{mg}, 0.2 \mathrm{mmol})$ and 2a (88.9 mg, $0.22 \mathrm{mmol}, 1.1$ equiv.). The crude product was purified by column chromatography (Wakogel ${ }^{\circledR}$ C-200, 19:1 toluene/EtOAc) to provide 8k (29.0 mg, 46\%, as a white solid) and 4-nitro- $N$-(1-phenylprop-2-enyl)benzenesulfonamide $(\mathbf{9})^{20}(8.2 \mathrm{mg}, 13 \%$ as a pale yellow oil).

8k: $R_{f}=0.44\left(9: 1\right.$ toluene/EtOAc); mp $113.0-115.0^{\circ} \mathrm{C} ;[\alpha]_{\mathrm{D}}{ }^{23}-4.43^{\circ}\left(c 0.81\right.$, acetone) for $56 \%$ ee [lit. ${ }^{24}$ $[\alpha]_{\mathrm{D}}{ }^{25}-10^{\circ}$ (c 0.25, acetone) for $\left.(S)-8 \mathrm{k}\right] ;{ }^{1} \mathrm{H}$ NMR $\left(400 \mathrm{MHz}, \mathrm{CDCl}_{3}\right) \delta 2.32(\mathrm{~d}, J=4.5 \mathrm{~Hz}, 1 \mathrm{H}, \mathrm{NCHH})$, $2.47(\mathrm{dd}, J=8.2,14.2 \mathrm{~Hz}, 1 \mathrm{H}, \mathrm{NCH}), 2.90(\mathrm{~d}, J=6.9 \mathrm{~Hz}, \mathrm{PhCH} H), 2.96-3.05(\mathrm{~m}, 2 \mathrm{H}, \mathrm{NCH} H$ and $\mathrm{PhCHH}), 6.96-6.98$ (m, 2H, Ar), 7.06-7.14 (m, 3H, Ar), 7.88 (d, J=8.6 Hz, 2H, Ar), 8.16 (d, J = 8.6 Hz, $2 \mathrm{H}, A r)$. The enantiomeric excess of $\mathbf{8 k}$ was determined to be $56 \%$ by HPLC with a Chiralpak AD-H column $(3: 1$ hexane $/ i-\mathrm{PrOH}, 1.0 \mathrm{~mL} / \mathrm{min}): t_{\mathrm{R}}($ minor $)=11.7 \mathrm{~min}$ for $(R)$-enantiomer; $t_{\mathrm{R}}($ major $)=12.8$ $\min$ for $(S)$-enantiomer.

9: $R_{f}=0.24\left(9: 1\right.$ toluene/EtOAc); $[\alpha]_{\mathrm{D}}{ }^{23}-4.63^{\circ}\left(c 0.39, \mathrm{CHCl}_{3}\right)$ for $20 \%$ ee; ${ }^{1} \mathrm{H} \mathrm{NMR}\left(400 \mathrm{MHz}, \mathrm{CDCl}_{3}\right)$ d 5.08-5.21 (m, 4H, NH, PhCH and $\left.=\mathrm{CH}_{2}\right), 5.89\left(\mathrm{ddd}, J=5.8,10.5,16.3 \mathrm{~Hz}, 1 \mathrm{H}, \mathrm{CH}_{2}=\mathrm{CH}\right), 7.07-7.09(\mathrm{~m}$, 2H, Ar), 7.19-7.21 (m, 3H, Ar), $7.84(\mathrm{dt} J=8.7 \mathrm{~Hz}, 2 \mathrm{H}, A r), 8.18(\mathrm{~d}, J=8.7 \mathrm{~Hz}, 2 \mathrm{H}, A r)$. The enantiomeric excess of 9 was determined to be $14 \%$ by HPLC with a Chiralpak AD-H column (3:1 
hexane $/ i-\mathrm{PrOH}, 1.0 \mathrm{~mL} / \mathrm{min}): t_{\mathrm{R}}($ minor $)=7.9 \mathrm{~min} ; t_{\mathrm{R}}$ (major) $=8.9 \mathrm{~min}$. The absolute configuration of 9 was not determined.

1,1a,6,6a-Tetrahydro-1-(4-nitrophenylsulfonyl)-indeno[1,2-b]azirine (81). ${ }^{1 \mathrm{~b}}$ According to the general procedure for enantioselective aziridination reaction, $8 \mathbf{1}$ was prepared from indene (7j) (23.2 $\mathrm{mg}, 0.2$ mmol) and 2a (88.9 $\mathrm{mg}, 0.22 \mathrm{mmol}, 1.1$ equiv.). The crude product was purified by column chromatography (Wakogel ${ }^{\circledR} \mathrm{C}-200,9: 1$ hexane/EtOAc) to provide 81 (34.0 $\mathrm{mg}, 54 \%$ ) as a pale yellow solid; $R_{f}=0.43(3: 1$ hexane/EtOAc $) ; \mathrm{mp}=126.0-127.0{ }^{\circ} \mathrm{C} ;[\alpha]_{\mathrm{D}}{ }^{23}+4.68^{\circ}\left(c 1.07, \mathrm{CHCl}_{3}\right)$ for $15 \%$ ee; ${ }^{1} \mathrm{H}$ NMR (400 MHz, $\left.\mathrm{CDCl}_{3}\right) \delta 3.15(\mathrm{dd}, J=0.9,17.9 \mathrm{~Hz}, 1 \mathrm{H}, \operatorname{ArCH} H), 3.22(\mathrm{dd}, J=4.2,17.9 \mathrm{~Hz}, 1 \mathrm{H}$, $\operatorname{ArCHH}), 4.04\left(\mathrm{ddd}, J=0.9,4.2,5.1 \mathrm{~Hz}, 1 \mathrm{H}, \mathrm{NCHCH}_{2}\right), 4.41(\mathrm{~d}, J=5.1 \mathrm{~Hz}, 1 \mathrm{H}, \operatorname{ArCHN}), 7.18-7.42(\mathrm{~m}$, 4H, $A r), 8.14(\mathrm{~d}, J=9.0 \mathrm{~Hz}, 2 \mathrm{H}, A r), 8.36(\mathrm{~d}, J=9.0 \mathrm{~Hz}, 2 \mathrm{H}, A r)$. The enantiomeric excess of $8 \mathbf{l}$ was determined to be $15 \%$ by HPLC with a Chiralpak AD-H column $(9: 1$ hexane $/ i-\mathrm{PrOH}, 1.0 \mathrm{~mL} / \mathrm{min}): t_{\mathrm{R}}$ $($ major $)=34.7 \mathrm{~min} ; t_{\mathrm{R}}($ minor $)=37.3 \mathrm{~min}$. The absolute configuration of $\mathbf{8 1}$ was not determined.

1a,2,3,7,7b-Tetrahydro-1-(4-nitrophenylsulfonyl)-1H-naphth[1,2-b]azirine $(\mathbf{8 m}) .{ }^{25}$ According to the general procedure for enantioselective aziridination reaction, $\mathbf{8 m}$ was prepared from 1,2-dihydronaphthalene (7k) (26.0 mg, $0.2 \mathrm{mmol})$ and $\mathbf{2 a}(88.9 \mathrm{mg}, 0.22 \mathrm{mmol}, 1.1$ equiv.). The crude product was purified by column chromatography (Wakoge ${ }^{\circledR}$ C-200, 9:1 hexane/EtOAc) to provide $\mathbf{8 m}$ $(53.7 \mathrm{mg}, 83 \%)$ as a white solid; $R_{f}=0.46(3: 1$ hexane $/$ EtOAc $) ; \mathrm{mp}=136.0-137.5^{\circ} \mathrm{C} ;[\alpha]_{\mathrm{D}}^{23}-22.0^{\circ}(c$ 0.88, $\left.\mathrm{CHCl}_{3}\right)$ for $32 \%$ ee; ${ }^{1} \mathrm{H} \mathrm{NMR}\left(400 \mathrm{MHz}, \mathrm{CDCl}_{3}\right) \delta 1.75(\mathrm{~m}, 1 \mathrm{H}, \mathrm{NCHCH}), 2.31(\mathrm{~m}, 1 \mathrm{H}$, $\mathrm{NCHCHH}), 2.59$ (dd, $J=5.5,14.8 \mathrm{~Hz}, 1 \mathrm{H}, \mathrm{ArCH} H), 2.73(\mathrm{ddd}, J=6.1,14.8,14.8 \mathrm{~Hz}, 1 \mathrm{H}, \operatorname{ArCHH}), 3.72$ $(\mathrm{d}, J=7.1 \mathrm{~Hz}, 1 \mathrm{H}, \mathrm{NCHCH}), 3.93$ (d, $J=7.1 \mathrm{~Hz}, 1 \mathrm{H}, \mathrm{NCHAr}), 7.06-7.31$ (m, 4H, Ar), 8.12 (d, $J=9.2$ $\mathrm{Hz}, 2 \mathrm{H}, A r), 8.35(\mathrm{~d}, J=9.2 \mathrm{~Hz}, 2 \mathrm{H}, A r)$. The enantiomeric excess of $\mathbf{8 m}$ was determined to be $32 \%$ by HPLC with a Chiralpak AS-H column $(3: 1$ hexane $/ i-\mathrm{PrOH}, 1.0 \mathrm{~mL} / \mathrm{min}): t_{\mathrm{R}}($ major $)=28.5 \mathrm{~min} ; t_{\mathrm{R}}$ (minor) $=38.6 \mathrm{~min}$. The absolute configuration of $\mathbf{8} \mathbf{m}$ was not determined.

\section{1,1a,2,7b-Tetrahydro-2,2-dimethyl-1-(4-nitrophenylsulfonyl)-[1]benzopyrano[3,4-b]azirine}

(8n).

According to the general procedure for enantioselective aziridination reaction, 8n was prepared from 2,2-dimethyl-2H-chromene (7l) $(16.0 \mathrm{mg}, 0.1 \mathrm{mmol})$ and $\mathbf{2 a}$ (44.5 mg, $0.11 \mathrm{mmol}, 1.1$ equiv.). The crude product was purified by column chromatography (Wakoge ${ }^{\circledR}$ C-200, 9:1 hexane/EtOAc) to provide 8n (35.6 mg, 98\%) as a colorless oil; $R_{f}=0.46\left(3: 1\right.$ hexane/EtOAc); $[\alpha]_{\mathrm{D}}^{23}+13.1^{\circ}\left(c 1.05, \mathrm{CHCl}_{3}\right)$ for $94 \%$ ee; IR(neat) v: 1531, 1350, $1163 \mathrm{~cm}^{-1} ;{ }^{1} \mathrm{H}$ NMR (400 MHz, $\left.\mathrm{CDCl}_{3}\right) \delta 1.26\left(\mathrm{~s}, 3 \mathrm{H}, \mathrm{CH}_{3}\right), 1.36\left(\mathrm{~s}, 3 \mathrm{H}, \mathrm{CH}_{3}\right)$, $3.51(\mathrm{~d}, J=7.5 \mathrm{~Hz}, 1 \mathrm{H}, \mathrm{NCH}), 4.02(\mathrm{~d}, J=7.5 \mathrm{~Hz}, 1 \mathrm{H}, \mathrm{ArCH}), 6.78(\mathrm{~d}, J=8.1 \mathrm{~Hz}, 1 \mathrm{H}, A r), 6.92(\mathrm{t}, J=$ $7.4 \mathrm{~Hz}, 1 \mathrm{H}, A r), 7.21-7.28(\mathrm{~m}, 2 \mathrm{H}, A r), 8.16(\mathrm{~d}, J=8.7 \mathrm{~Hz}, 2 \mathrm{H}, A r), 8.36(\mathrm{~d}, J=8.7 \mathrm{~Hz}, 2 \mathrm{H}, A r) ;{ }^{13} \mathrm{C}$ 
NMR (100 MHz, CDCl $) \delta 23.22\left(\mathrm{CH}_{3}\right), 26.12\left(\mathrm{CH}_{3}\right), 41.45(\mathrm{CH}), 50.83(\mathrm{CH}), 71.58(\mathrm{C}), 117.11(\mathrm{C})$, $118.43(\mathrm{CH}), 121.66(\mathrm{CH}), 124.30(\mathrm{CH}), 129.18(\mathrm{CH}), 129.22(\mathrm{CH}), 130.72(\mathrm{CH}), 143.87(\mathrm{C}), 150.60(\mathrm{C})$, 152.43 (C); HRMS (EI) calcd for $\mathrm{C}_{17} \mathrm{H}_{16} \mathrm{~N}_{2} \mathrm{O}_{5} \mathrm{~S}\left(\mathrm{M}^{+}\right)$360.0780, found 360.0785. The enantiomeric excess of 8n was determined to be $94 \%$ by HPLC with a Chiralcel OD-H column $(9: 1$ hexane/i-PrOH, 0.5 $\mathrm{mL} / \mathrm{min}): t_{\mathrm{R}}($ minor $)=28.2 \mathrm{~min} ; t_{\mathrm{R}}($ major $)=31.4 \mathrm{~min}$. The absolute configuration of $\mathbf{8 n}$ was not determined.

\section{ACKNOWLEDGEMENTS}

This research was supported, in part, by a Grant-in-Aid for Scientific Research on Priority Areas "Advanced Molecular Transformations of Carbon Resources" from the Ministry of Education, Culture, Sports, Science and Technology, Japan. We thank S. Oka, M. Kiuchi and T. Hirose of the Center for Instrumental Analysis at Hokkaido University for mass measurements.

\section{REFERENCES AND NOTES}

1. For recent reviews on aziridination of alkenes with iminoiodinanes, see: (a) P. Müller, 'Advances in Catalytic Processes,' ed. by M. P. Doyle, JAI Press, Greenwich, 1997, Volume 2, 113. (b) E. N. Jacobsen, 'Comprehensive Asymmetric Catalysis,' ed. by E. N. Jacobsen, A. Pfaltz, and H. Yamamoto, Springer, Berlin, 1999, Vol. 2, Chapter 17. (c) T. Katsuki, 'Catalytic Asymmetric Synthesis,' ed. by I. Ojima, Wiley-VCH, New York, 2000, Chapter 6B4. (d) P. Dauban and R. H. Dodd, Synlett, 2003, 1571. (e) M. P. Doyle, 'Reactive Intermediate Chemistry,' ed. by R. A. Moss, M. S. Platz, and M. Jones, Jr., John Wiley \& Sons, Hoboken, 2004, Chapter 12. (f) P. Müller and C. Fruit, Chem. Rev., 2003, 103, 2905.

2. (a) D. A. Evans, K. A. Woerpel, M. M. Hinman, and M. M. Faul, J. Am. Chem. Soc., 1991, 113, 726.

(b) R. E. Lowenthal and S. Masamune, Tetrahedron Lett., 1991, 32, 7373. (c) D. A. Evans, M. M. Faul, M. T. Bilodeau, B. A. Anderson, and D. M. Barnes, J. Am. Chem. Soc., 1993, 115, 5328. (d) M. J. Södergren, D. A. Alonso, and P. G. Andersson, Tetrahedron: Asymmetry, 1997, 8, 3563. (e) P. Dauban, L. Saniére, A. Tarrade, and R. H. Dodd, J. Am. Chem. Soc., 2001, 123, 7707. (f) J. Xu, L. Ma, and P. Jiao, Chem. Commun., 2004, 1616. (g) H.-L. Kwong, D. Liu, K.-Y. Chan, C.-S. Lee, K.-H. Huang, and C.-M. Che, Tetrahedron Lett., 2004, 45, 3965. (h) L. Ma, P. Jiao, Q. Zhang, and J. $\mathrm{Xu}$, Tetrahedron: Asymmetry, 2005, 16, 3718.

3. (a) Z. Li, K. R. Conser, and E. N. Jacobsen, J. Am. Chem. Soc., 1993, 115, 5326. (b) Z. Li, R. W. Quan, and E. N. Jacobsen, J. Am. Chem. Soc., 1995, 117, 5889. (c) D.-J. Cho, S.-J. Jeon, H.-S. Kim, C.-S. Cho, S.-C. Shim, and T.-J. Kim, Tetrahedron: Asymmetry, 1999, 10, 3833. (d) C. J. Sanders, K. 
M. Gillespie, D. Bell, and P. Scott, J. Am. Chem. Soc., 2000, 122, 7132. (e) K. M. Gillespie, E. J. Crust, R. J. Deeth, and P. Scott, Chem. Commun., 2001, 785. (f) M. Shi, C.-J. Wang, and A. S. C. Chan, Tetrahedron: Asymmetry, 2001, 12, 3105. (g) K. M. Gillespie, C. J. Sanders, P. O'Shaughnessy, I. Westmoreland, C. P. Thickitt, and P. Scott. J. Org. Chem., 2002, 67, 3450. (h) H. Suga, A. Kakehi, S. Ito, T. Ibata, T. Fudo, Y. Watanabe, and Y. Kinoshita, Bull. Chem. Soc. Jpn., 2003, 76, 189. (i) X. Wang and K. Ding, Chem. Eur. J., 2006, 12, 4568.

4. (a) D. Tanner, P. G. Andersson, A. Harden, and P. Somfai, Tetrahedron Lett., 1994, 35, 4631. (b) D. B. Llewellyn, D. Adamson, and B. A. Arndtsen, Org. Lett., 2000, 2, 4165. (c) T. C. H. Lam, W.-L. Mak, W.-L. Wong, H.-L. Kwong, H. H. Y. Sung, S. M. F. Lo, I. D. Williams, and W.-H. Leung, Organometallics, 2004, 23, 1247.

5. (a) K. Noda, N. Hosoya, R. Irie, Y. Ito, and T. Katsuki, Synlett, 1993, 469. (b) H. Nishikori and T. Katsuki, Tetrahedron Lett., 1996, 37, 9245.

6. (a) T.-S. Lai, H.-L. Kwong, C.-M. Che, and S.-M. Peng, Chem. Commun., 1997, 2373. (b) J.-P. Simonato, J. Pécaut, W. R. Scheidt, and J.-C. Marchon, Chem. Commun., 1999, 989. (c) J.-L. Liang, J.-S. Huang, X.-Q. Yu, N. Zhu, and C.-M. Che, Chem. Eur. J., 2002, 8, 1563.

7. J.-L. Liang, X.-Q. Yu, and C.-M. Che, Chem. Commun., 2002, 124.

8. Chiral ruthenium(II) salen complexes of Katsuki have recently been reported to catalyze the aziridination of various terminal conjugated alkenes using sulfonyl azides as a nitrene precursor with excellent enantioselectivity and turnover number: (a) K. Omura, M. Murakami, T. Uchida, R. Irie, and T. Katsuki, Chem. Lett., 2003, 32, 354. (b) K. Omura, T. Uchida, R. Irie, and T. Katsuki, Chem. Commun., 2004, 2060. (c) T. Katsuki, Chem. Lett., 2005, 34, 1304. (d) H. Kawabata, K. Omura, and T. Katsuki, Tetrahedron Lett., 2006, 47, 1571.

9. R. Breslow and S. H. Gellman, J. Am. Chem. Soc., 1983, 105, 6728.

10. (a) D. A. Evans, M. M. Faul, and M. T. Bilodeau, J. Org. Chem., 1991, 56, 6744. (b) D. A. Evans, M. M. Faul, and M. T. Bilodeau, J. Am. Chem. Soc., 1994, 116, 2742.

11. (a) P. Müller, C. Baud, and Y. Jacquier, Tetrahedron, 1996, 52, 1543. (b) P. Müller, C. Baud, and Y. Jacquier, Can. J. Chem., 1998, 76, 738.

12. (a) J.-L. Liang, S.-X. Yuan, P. W. H. Chan, and C.-M. Che, Org. Lett., 2002, 4, 4507. (b) J.-L. Liang, S.-X. Yuan, P. W. H. Chan, and C.-M. Che, Tetrahedron Lett., 2003, 44, 5917. (c) C. Fruit and P. Müller, Tetrahedron: Asymmetry, 2004, 15, 1019. (d) C. J. Hayes, P. W. Beavis, and L. A. Humphries, Chem. Commun., 2006, 4501.

13. M. Yamawaki, H. Tsutsui, S. Kitagaki, M. Anada, and S. Hashimoto, Tetrahedron Lett., 2002, 43, 9561.

14. (a) N. Watanabe, T. Ogawa, Y. Ohtake, S. Ikegami, and S. Hashimoto, Synlett, 1996, 85. (b) M. 
Anada and S. Hashimoto, Tetrahedron Lett., 1998, 39, 79. (c) M. Anada and S. Hashimoto, Tetrahedron Lett., 1998, 39, 9063. (d) S. Kitagaki, M. Yasugahira, M. Anada, M. Nakajima, and S. Hashimoto, Tetrahedron Lett., 2000, 41, 5931. (e) T. Takahashi, H. Tsutsui, M. Tamura, S. Kitagaki, M. Nakajima, and S. Hashimoto, Chem. Commun., 2001, 1604. (f) S. Kitagaki, Y. Yanamoto, H. Tsutsui, M. Anada, M. Nakajima, and S. Hashimoto, Tetrahedron Lett., 2001, 42, 6361. (g) H. Tsutsui, M. Matsuura, K. Makino, S. Nakamura, M. Nakajima, S. Kitagaki, and S. Hashimoto, Isr. J. Chem., 2001, 41, 283. (h) H. Saito, H. Oishi, S. Kitagaki, S. Nakamura, M. Anada, and S. Hashimoto, Org. Lett., 2002, 4, 3887. (i) K. Minami, H. Saito, H. Tsutsui, H. Nambu, M. Anada, and S. Hashimoto, Adv. Synth. Catal., 2005, 347, 1483. (j) Y. Natori, M. Anada, S. Nakamura, H. Nambu, and S. Hashimoto, Heterocycles, 2006, 70, 635.

15. For a practical synthesis of $\mathrm{Rh}_{2}(\mathrm{~S} \text {-PTTL })_{4}$, see: H. Tsutsui, T. Abe, S. Nakamura, M. Anada, and $\mathrm{S}$. Hashimoto, Chem. Pharm. Bull., 2005, 53, 1366.

16. M. Yamawaki, S. Kitagaki, M. Anada, and S. Hashimoto, Heterocycles, 2006, 69, 527.

17. J. Farrás, X. Ginesta, P. W. Sutton, J. Taltavull, F. Egeler, P. Romea, F. Urpí, and J. Vilarrasa, Tetrahedron, 2001, 57, 7665.

18. The reaction of 7a with [ $N$-(4-methyphenylsulfony)imino]phenyliodinane in the presence of 2 mol \% of $\mathbf{1 a}\left(\mathrm{CH}_{2} \mathrm{Cl}_{2}, 0^{\circ} \mathrm{C}\right)$ gave a complex mixture of products.

19. H. Tsutsui, Y. Yamaguchi, S. Kitagaki, S. Nakamura, M. Anada, and S. Hashimoto, Tetrahedron: Asymmetry, 2003, 14, 817.

20. I. Nägeli, C. Baud, G. Bernardinelli, Y. Jacquier, M. Moran, and P. Müller, Helv. Chim. Acta, 1997, 80, 1087.

21. (a) Y. Yamada, T. Yamamoto, and M. Okawara, Chem. Lett., 1975, 4, 361. (b) M. J. Södergren, D. A. Alonso, A. V. Bedekar, and P. G. Andersson, Tetrahedron Lett., 1997, 38, 6897.

22. S. K. Kim and E. N. Jacobsen, Angew. Chem. Int. Ed., 2004, 43, 3952.

23. S. K.-Y. Leung, W.-M. Tsui, J.-S. Huang, C.-M. Che, J.-L. Liang, and N. Zhu, J. Am. Chem. Soc., $2005,127,16629$.

24. D. A. Alonso and P. G. Andersson, J. Org. Chem., 1998, 63, 9455.

25. G. F. Keaney and J. L. Wood, Tetrahedron Lett., 2005, 46, 4031. 